\title{
FGMOS BASED LOW-VOLTAGE LOW-POWER HIGH OUTPUT IMPEDANCE REGULATED CASCODE CURRENT MIRROR
}

\author{
Abhinav Anand ${ }^{1}$, Prof. Sushanta K. Mandal ${ }^{2}$, Anindita Dash ${ }^{3}$, B. Shivalal Patro ${ }^{4}$ \\ ${ }^{1,2,3,4}$ School of Electronics Engineering, KIIT University, Bhubaneswar, India \\ abhinavvlsi@gmail.com ${ }^{1}$, sushantakumar@yahoo.com ${ }^{2}$, litun.1103@gmail.com ${ }^{3}$, \\ shivalal.lali@gmail. $\mathrm{com}^{4}$
}

\begin{abstract}
Floating Gate MOS (FGMOS) transistors can be very well implemented in lieu of conventional MOSFET for design of a low-voltage, low-power current mirror. Incredible features of flexibility, controllability and tunability of FGMOS yields better results with respect to power, supply voltage and output swing. This paper presents a new current mirror designed with FGMOS which exhibit high output impedance, higher current range, very low power dissipation and higher matching accuracy. It achieves current range of up to $1500 \mu \mathrm{A}$, high output impedance of $1.125 \mathrm{T \Omega}$, bandwidth of $4.1 \mathrm{MHz}$ and dissipates power as low as 10.56 $\mu W$. The proposed design has been simulated using Cadence Design Environment in $180 \mathrm{~nm}$ CMOS process technology with +1.0 Volt single power supply.
\end{abstract}

\section{KEYWORDS}

Floating Gate MOSFET, Current Mirror, Regulated Cascode, Low-Voltage \& Low-Power

\section{INTRODUCTION}

A current mirror (CM) is a circuit designed to copy a current through one active device by controlling the current in another active device of circuit, keeping the output current constant regardless of loading. The responsibilities of current mirror circuit are current amplification and to provide proper biasing to analog circuits. Due to very wide application of current mirror in low voltage low-power analog circuits, accuracy, output impedance and power consumption play key role in determining CM performance [6]. Their utilization in analog signal processing extends the advantages of low-voltage operations, derivation of resistorless topologies and electronic adjustment capability of their frequency characteristics [13].

With the increasing demand for smaller and faster products, there is an ongoing trend in fabrication process towards smaller transistors. Reducing feature size arises potential problem like power dissipation and dielectric breakdown due to high electric field across the feature. These potential problems can be compensated by reducing the voltage and hence low-voltage power supply is beneficial. In addition to this battery lifetime is crucial factor in various applications and hence low-power device is also a compulsory requirement as per current market trend. In the quest to achieve low-voltage and low-power, various techniques have evolved in due course of time and Floating Gate MOS (FGMOS) technique is one amongst them. 
International Journal of VLSI design \& Communication Systems (VLSICS) Vol.4, No.2, April 2013

FGMOS is proven technology in digital circuits but has also gained pace when it comes to its application in the area of low-voltage, low-power analog design. FGMOS is a device in which the second gate generally called floating gate is electrically isolated but capacitively coupled to input gates. A control voltage present at one of the multi-input FGMOS and facility to additional weighted inputs provides wide range of tunability to the circuit [1]. Implementation of FGMOS allows threshold voltage $\left(\mathrm{V}_{\mathrm{th}}\right)$ controllability without reducing the feature size, thus operates at power supply voltage levels which are well below the intended operational limit. Also, it consumes less power than the minimum required power for a circuit designed with conventional MOSFET. Using regulated cascode configuration achieves high output impedance of the order of $g_{m}{ }^{2} r_{\text {out }}{ }^{3}$ where $g_{m}$ is device trans-conductance and $r_{\text {out }}$ is output resistance [5], [10]-[11].

Various FGMOS based current mirror designs have been presented in [4], [7] and [8]-[9]. FGMOS based current mirror design presented in [4] and [8] suffers from higher power dissipation and lower current range. On the other hand [7] and [9] both presents FGMOS based design having better results with respect to input impedance, bandwidth and supply voltage but exhibits very low output impedance. Current mirror suggested in [12] has ultra low power dissipation but at a very low biasing current of the order of atto ampere.

In this paper a new low-voltage, low-power regulated cascode current mirror has been proposed. At input side of the proposed design a 3-input FGMOS device has been introduced to control the input characteristics of $\mathrm{CM}$ by varying the input bias voltage at one of the floating gate. The design has been supported by the simulated results.

The rest of the paper is organised as follows: Brief introduction of FGMOS and regulated cascode current mirror (RCCM) is given in section 2. Section 3 describes the proposed low-voltage, lowPower CM (LV-LPCM). Simulation results are discussed in section 4. Finally, some conclusions are drawn in section 5 .

\section{FGMOS AND REgulated CASCODE CURRENT MirRoR}

In this section floating gate MOSFET (FGMOS) and regulated cascode CM (RCCM) have been briefly introduced with their diagrammatic representation. This section also justifies the implementation of FGMOS and RCCM into the final design.

\subsection{Floating Gate MOSFET (FGMOS)}

Floating Gate MOS transistors are widely used in digital world as EPROM, EEPROM, flash memories, and neuronal computational element in neural network, digital potentiometers and single transistor DAC's. But, it is also gaining its popularity in analog design and has found application in low-voltage low-power analog design and analog storage elements. A typical multi input floating gate transistor is shown in Figure 1. It is a conventional MOSFET in which the gate is capacitively coupled to the input using another poly-silicon layer. The equation that models the behaviour of floating gate voltage $\left(\mathrm{V}_{\mathrm{FG}}\right)$ of FGMOS is given by equation (1) [1], [3]. 


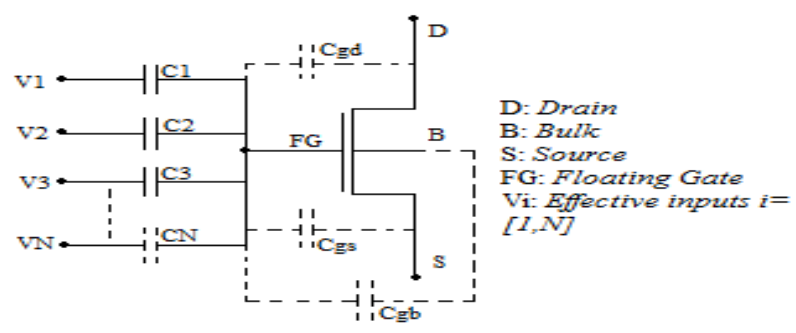

Figure 1. Equivalent schematic of $\mathrm{N}$-input n-channel FGMOS

$$
V_{F G}-\sum_{i=1}^{N} \frac{c_{i}}{c_{T}} V_{i}+\frac{c_{g s}}{c_{T}} V_{S}+\frac{c_{g A}}{c_{T}} V_{D}+\frac{Q_{F E}}{c_{T}}
$$

Where $c_{T}-c_{g s}+C_{g d}+C_{g b}+\Sigma_{i=1} C_{l}$ and $\mathrm{Q}_{\mathrm{FG}}$ accounts for the amount of charges that is being trapped in FG during fabrication.

FGMOS device provides impressive features relevant to low-voltage, low-power context. It shows flexibility in implementing both linear as well as non-linear functions. Independent control of threshold voltage $\left(\mathrm{V}_{\mathrm{th}}\right)$ accounts for controllability of the device. Also, since it is a multi-input device, FGMOS allows addition of extra inputs as per designer's requirement and hence provide tunability to the circuit.

In order to simulate FGMOS in Cadence design environment, a simulation model is needed which is shown in Figure 2 [1], [3]. In the model, the voltages $V_{1}, V_{2}, \ldots, V_{n}$ are the input voltages which are coupled to gate by resistor-capacitor parallel combination. $\mathrm{V}_{\mathrm{F}}$ and $\mathrm{V}_{0}$ are floating gate voltage and substrate voltage respectively. $\mathrm{M}$ is a conventional MOSFET.

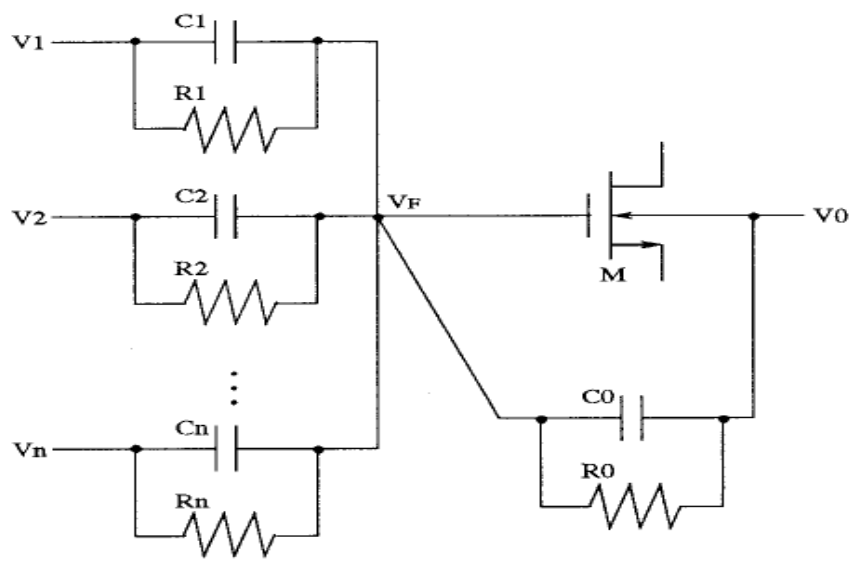

Figure 2. Simulation Model for N-input n-channel FGMOS 
International Journal of VLSI design \& Communication Systems (VLSICS) Vol.4, No.2, April 2013

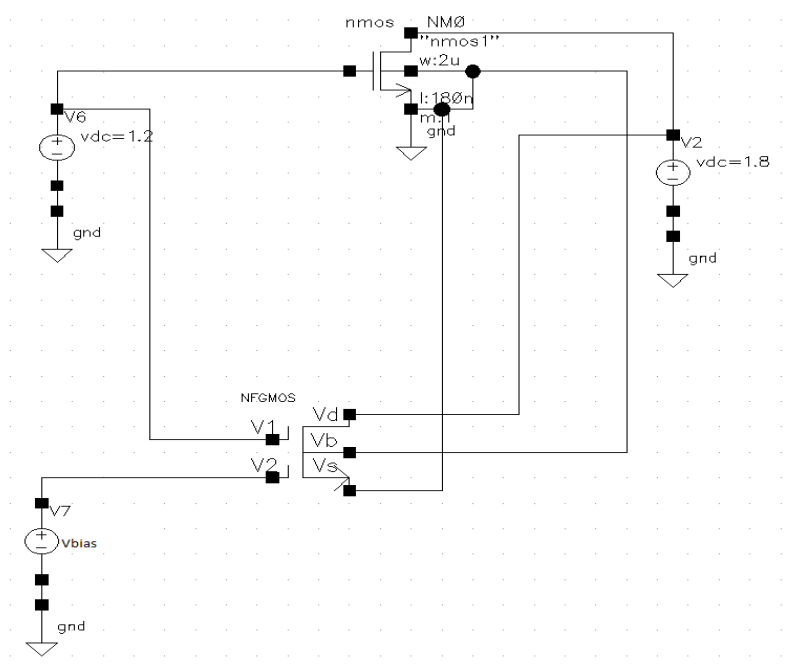

Figure 3. Test circuit for comparison between N-type FGMOS and NMOS

Figure 3 shows the test circuit for comparison between 2-input N-type FGMOS and a conventional NMOS. The simulated output characteristics and power dissipation on comparative basis is shown in Figure 4 and Figure 5 respectively.

DC Response

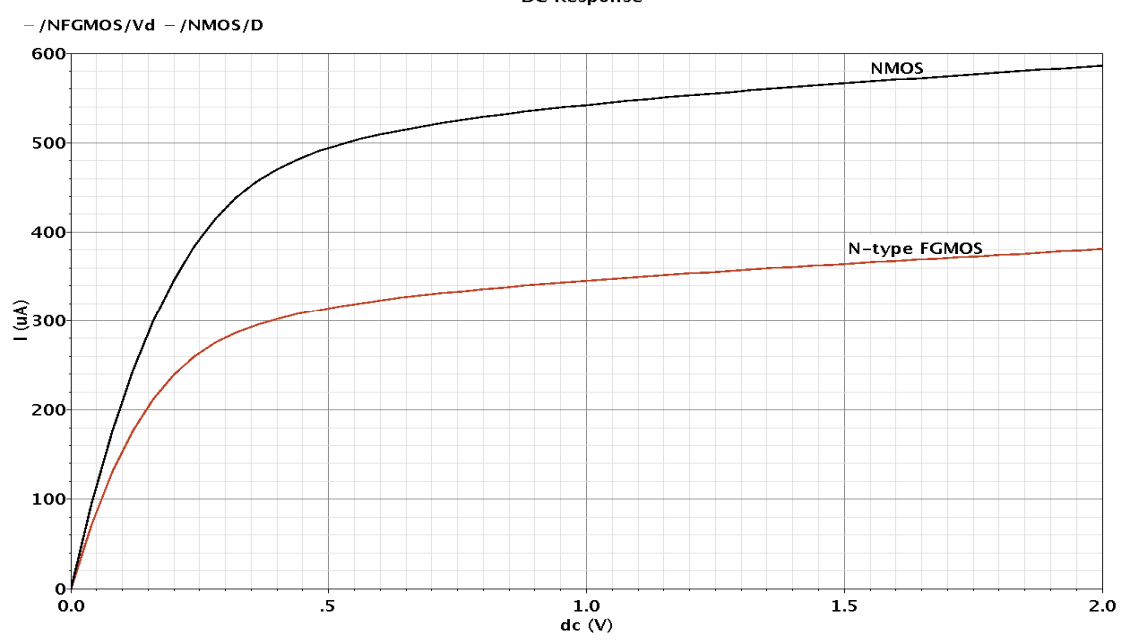

Figure 4. DC response of N-type FGMOS and conventional NMOS

Power dissipation of 2-input N-type FGMOS is $673.9 \mu \mathrm{W}$ for the test circuit, which is nearly $40 \%$ less than that of conventional NMOS transistor. This suffices for one of the reason why FGMOS device is used in low-voltage, low-power design. 
International Journal of VLSI design \& Communication Systems (VLSICS) Vol.4, No.2, April 2013
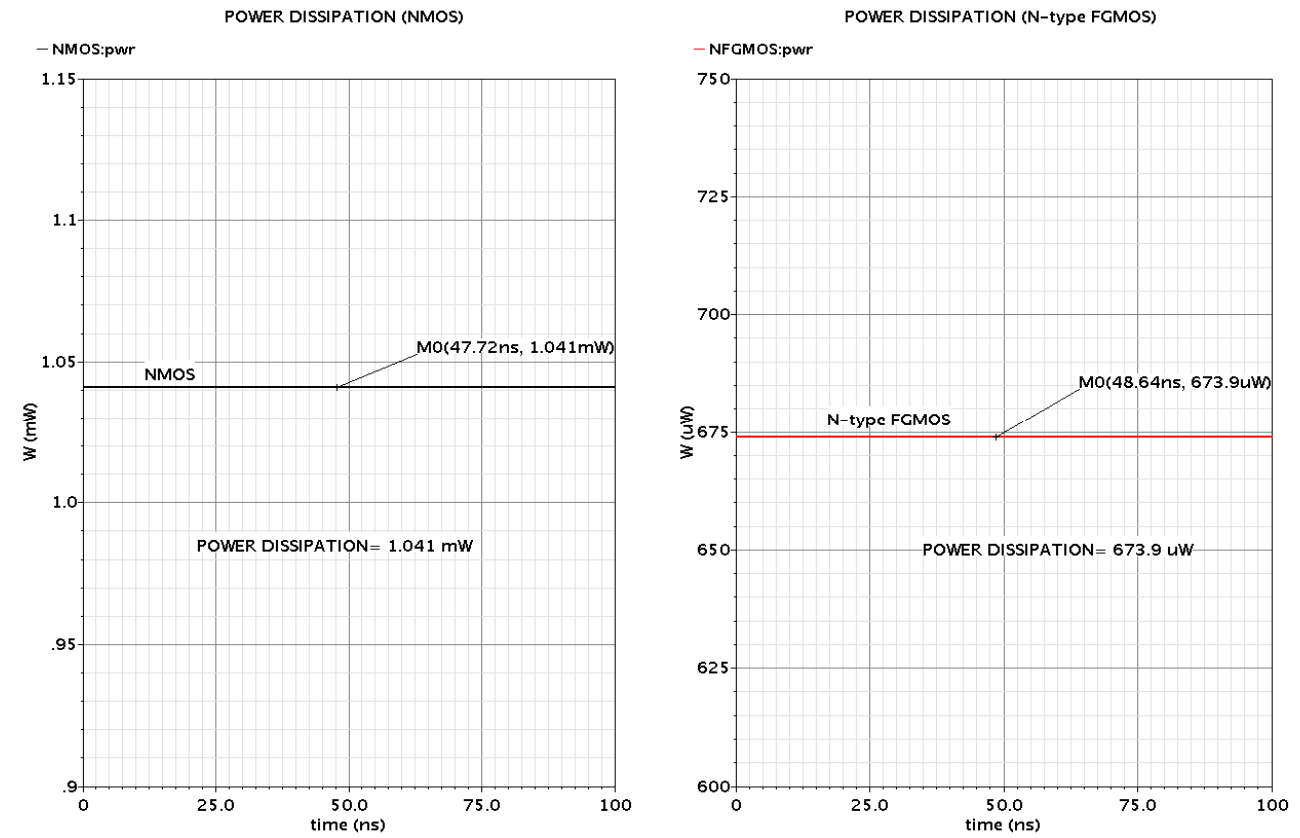

Figure 5. Power Dissipation of N-type FGMOS and conventional NMOS

\subsection{Regulated Cascode Current Mirror (RCCM)}

Regulated cascode CM is improved version of simple CM and it uses negative feedback concept for output current stabilization [2]. A typical regulated cascode configuration is shown in Figure 6. The primary reason behind considering RCCM configuration as a basis for the proposed design is its high output impedance of the order of $g_{m}{ }^{2} r_{\text {out }}{ }^{3}$. Also, current gain matching for regulated cascode configuration is good as long as primary transistors are properly biased.

\section{$V D D$}

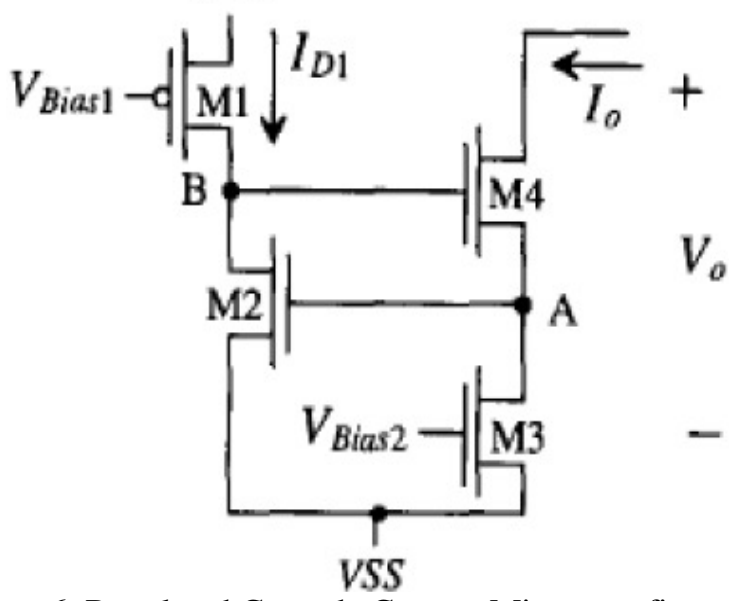

Figure 6. Regulated Cascode Current Mirror configuration 
International Journal of VLSI design \& Communication Systems (VLSICS) Vol.4, No.2, April 2013

\section{PROPOSED FGMOS BASED LV-LPCM}

The proposed LV-LP RCCM is based on FGMOS technique and is shown in Figure 7. It allows the design to work at a voltage as low as +0.8 Volts but works best at a single supply voltage of +1.0 Volts. It exhibits good matching accuracy and high output impedance of the order of 1.125 $\mathrm{T} \Omega$.

Table 1. Transistor Sizes of the Proposed CM

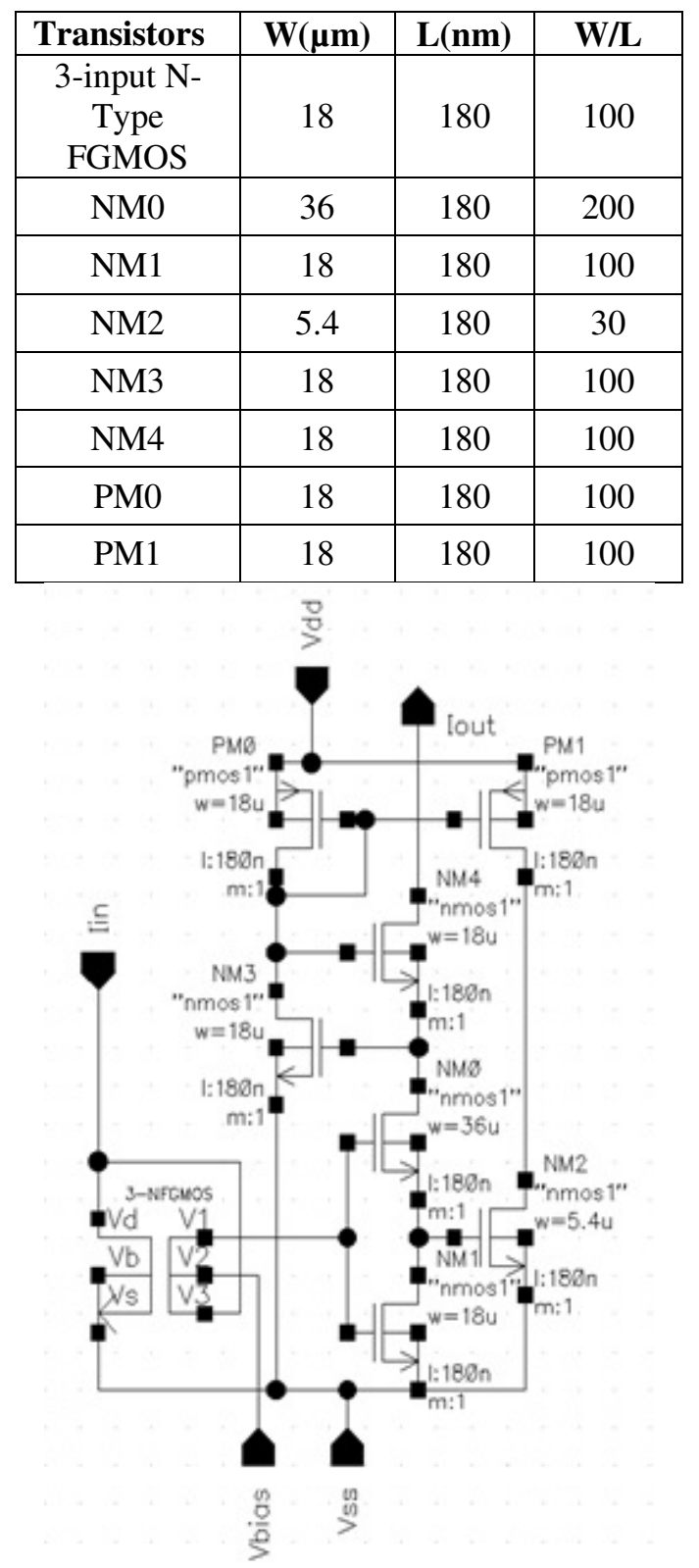

Figure 7. Proposed LV-LP Regulated Cascode CM 
Power dissipation of the circuit has been reduced to a value as low as $10.56 \mu \mathrm{W}$. It achieves higher current range of up to $1500 \mu \mathrm{A}$. The aspect ratios for various transistors have been summarized in Table1.

Transistors NM1, 3-input N-type FGMOS, NM4 and NM3 accounts for conventional regulated cascode CM. Transistors PM0 and PM1 are biasing transistors and provides accurate matching at the output. Transistor NM2 is connected in negative feedback to increase the output resistance and stabilize the output current. NM0 is specifically introduced in common gate configuration to achieve higher output resistance up to $\mathrm{T} \Omega$ range. The circuit however suffers from low bandwidth which is overshadowed by other satisfactory results viz. high output resistance, large current range and very low power dissipation.

\section{SIMULATION RESULTS}

The proposed circuit is simulated with Cadence Spectre simulator in Virtuoso ADEL environment. Various simulated results corresponding to its respective analysis viz. transient, DC or AC analysis with appropriate test circuits has been done. DC analysis is carried out to obtain current range. Figure 8 shows that the design achieves a current range of upto $1500 \mu \mathrm{A}$. Input and output characteristics are shown in Figure 9 and Figure 10 respectively.

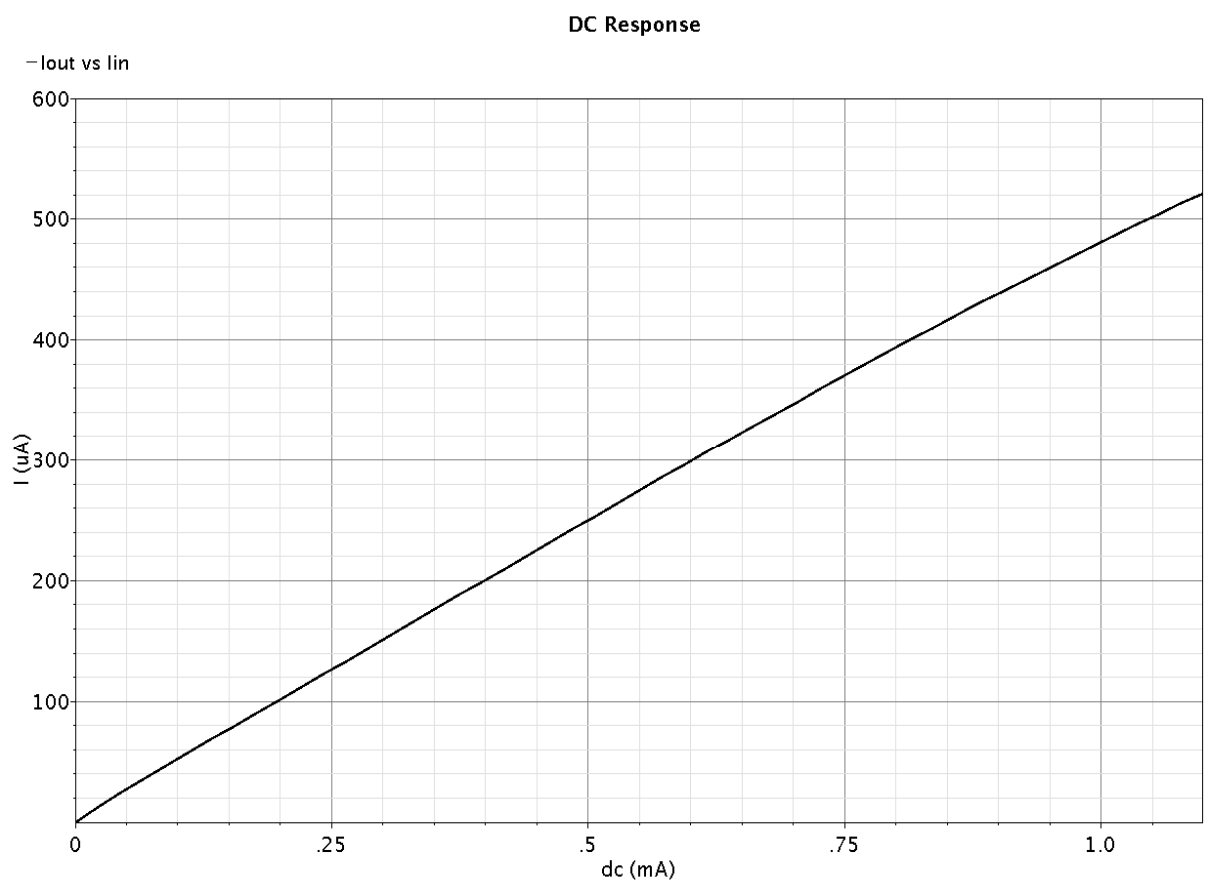

Figure 8. Transfer Characteristics 
International Journal of VLSI design \& Communication Systems (VLSICS) Vol.4, No.2, April 2013

INPUT CHARACTERISTICS

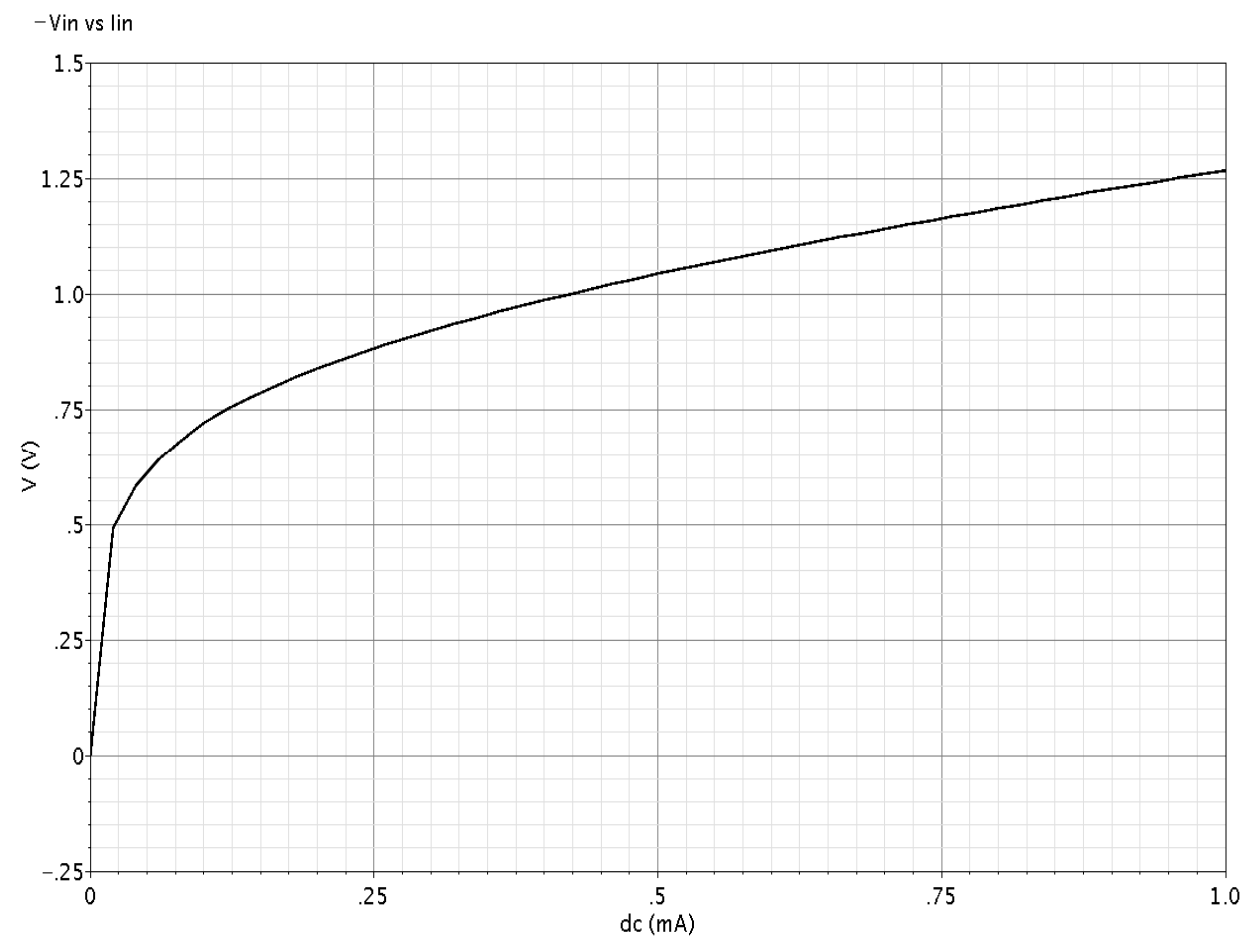

Figure 9. Input Characteristics OUTPUT CHARACTERISTICS

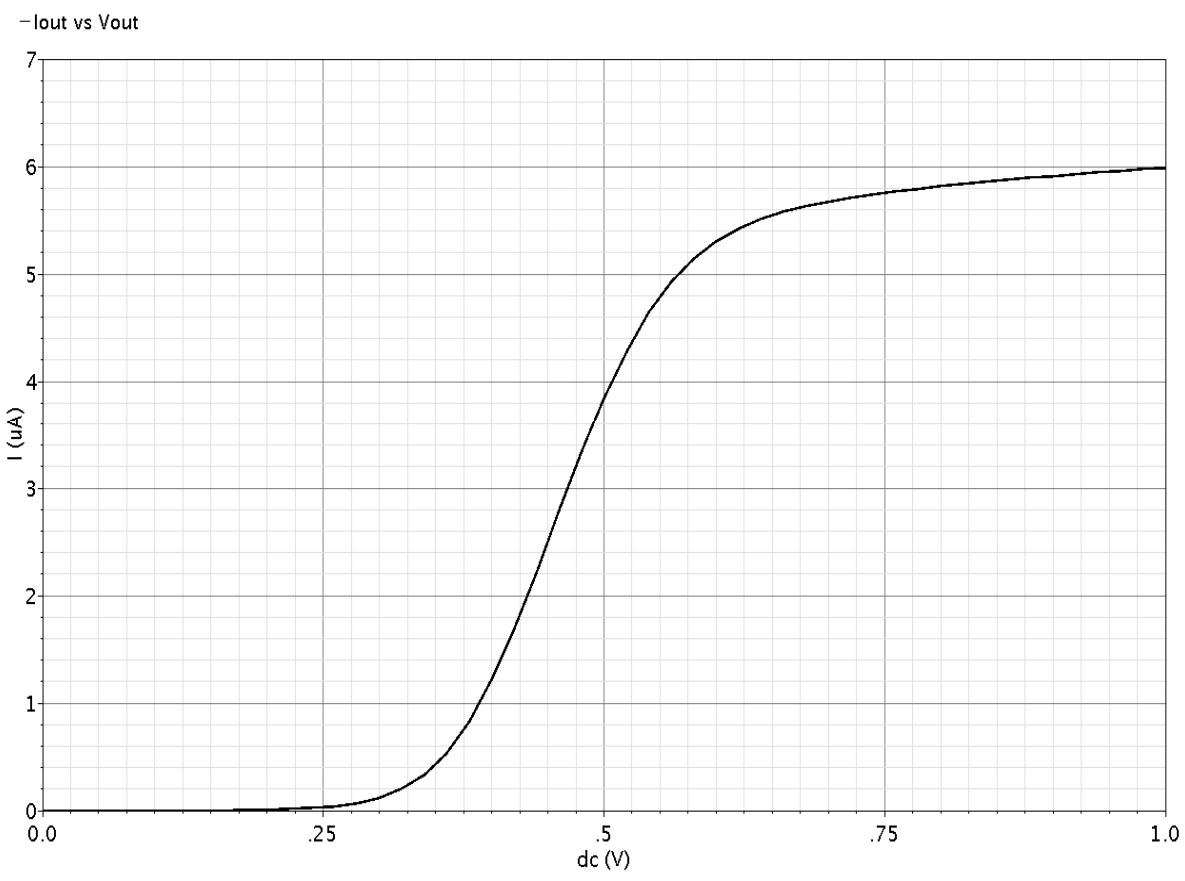

Figure 10. Output Characteristics 
International Journal of VLSI design \& Communication Systems (VLSICS) Vol.4, No.2, April 2013

Figure 11 illustrates the variation in input resistances with input biasing current. It can be observed from the graph that input impedance of the circuit is $24.5 \mathrm{~K} \Omega$ for biasing current of 20 $\mu \mathrm{A}$ and as low as $540 \Omega$ for a input biasing current of $500 \mu \mathrm{A}$.

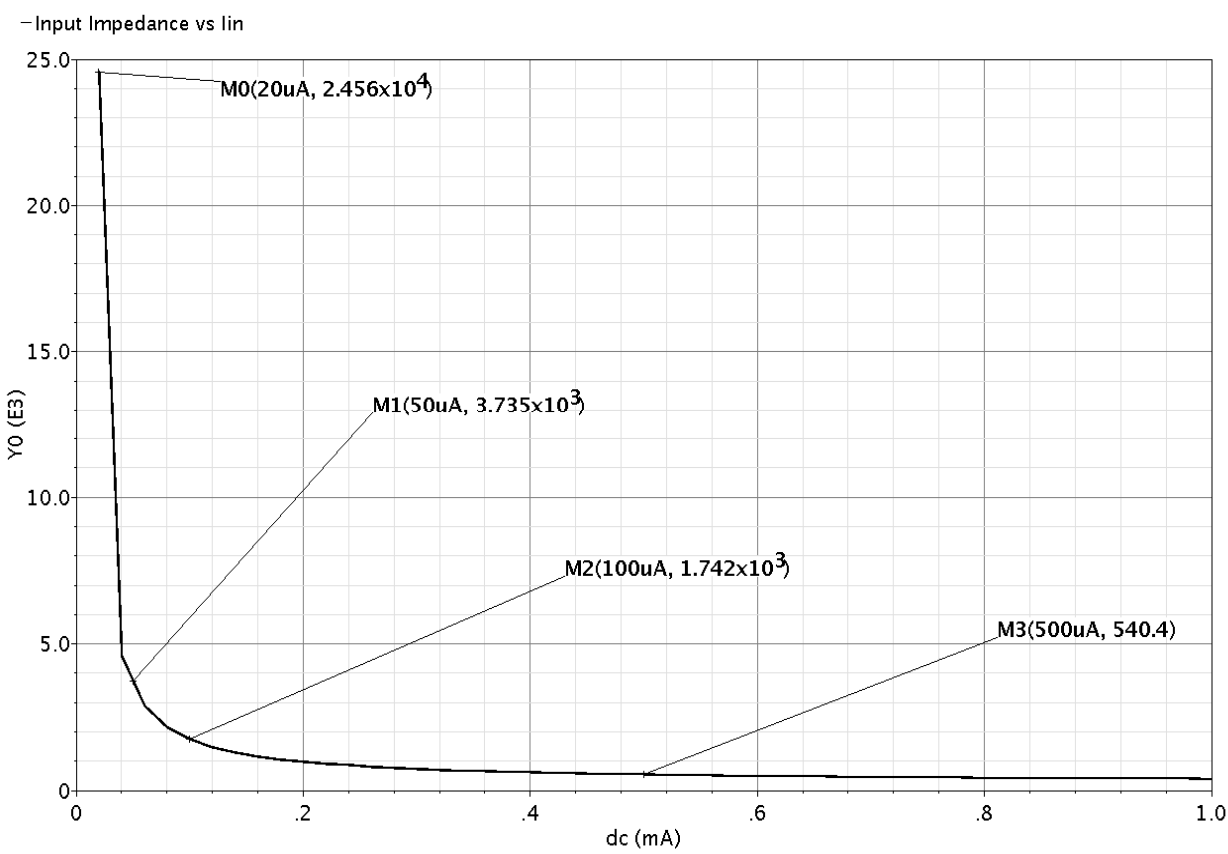

Figure 11. Input Impedance versus Input biasing current

Transient analysis is carried out to evaluate power dissipation of the circuit. It is observed to be $10.56 \mu \mathrm{W}$ as shown in Figure 12, which makes the circuit a low power design.

POWER DISSIPATION

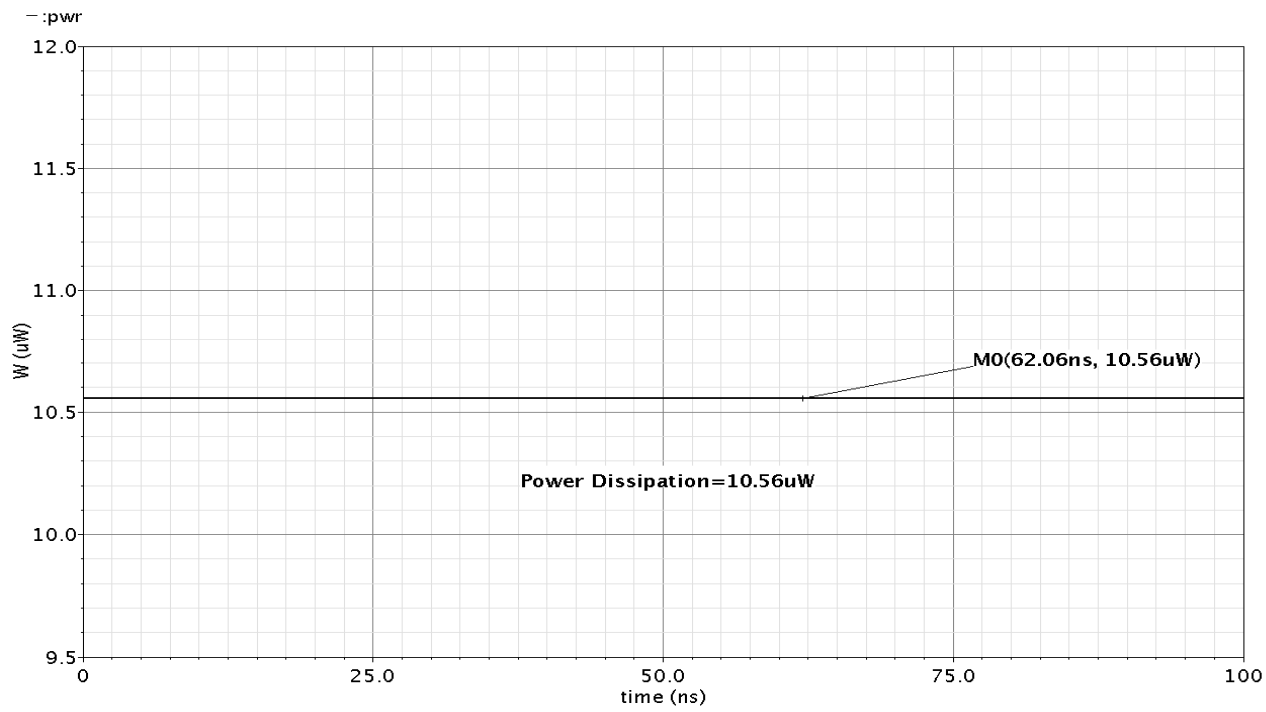

Figure 12. Power dissipation

AC analysis is performed to obtain the output impedance and bandwidth of the proposed FGMOS based LV-LP RCCM. The output impedance comes out to be $1.125 \mathrm{~T} \Omega$ and bandwidth of 4.1 $\mathrm{MHz}$ at a biasing current of $10 \mu \mathrm{A}$ and is shown in Figure 13 and Figure 14 respectively. 
International Journal of VLSI design \& Communication Systems (VLSICS) Vol.4, No.2, April 2013

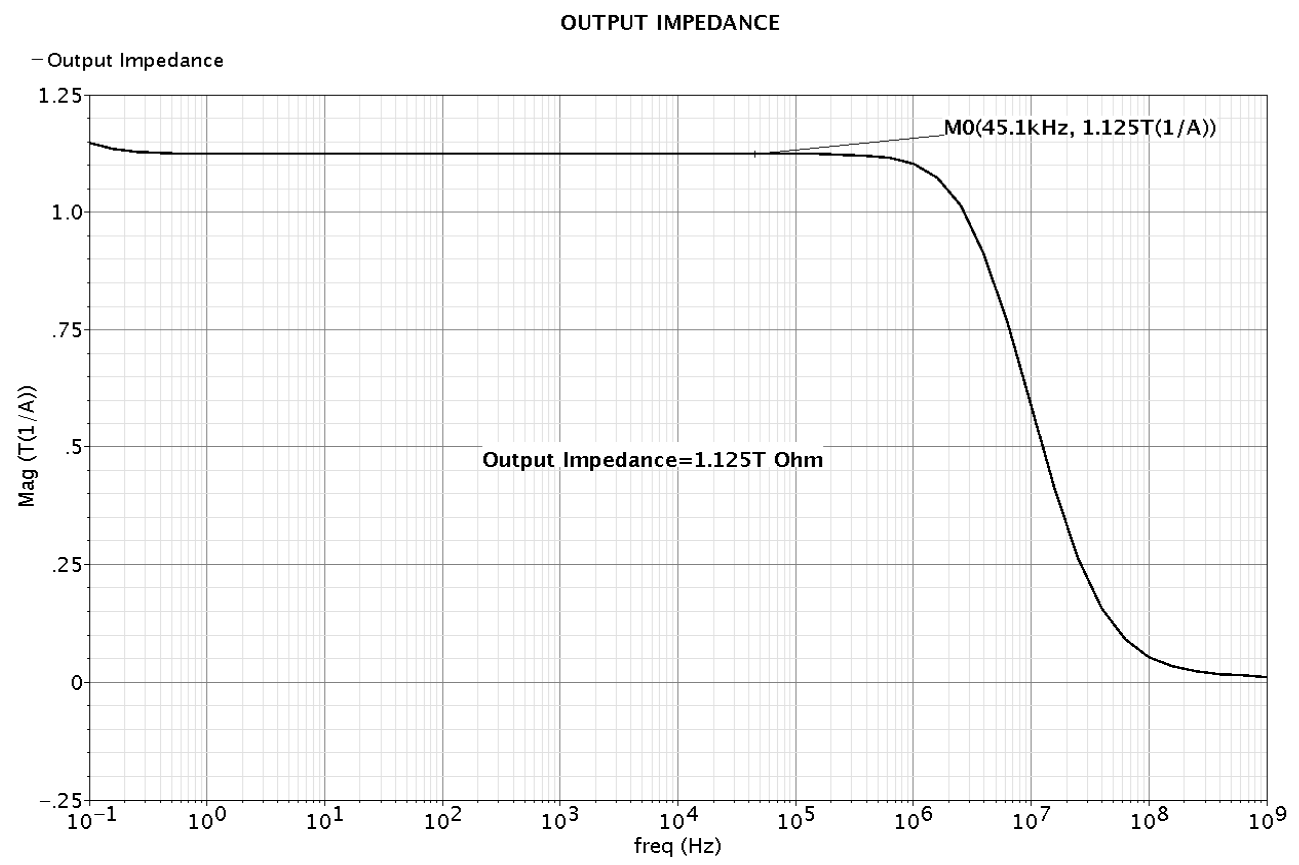

Figure 13. Output Impedance FREQUENCY RESPONSE

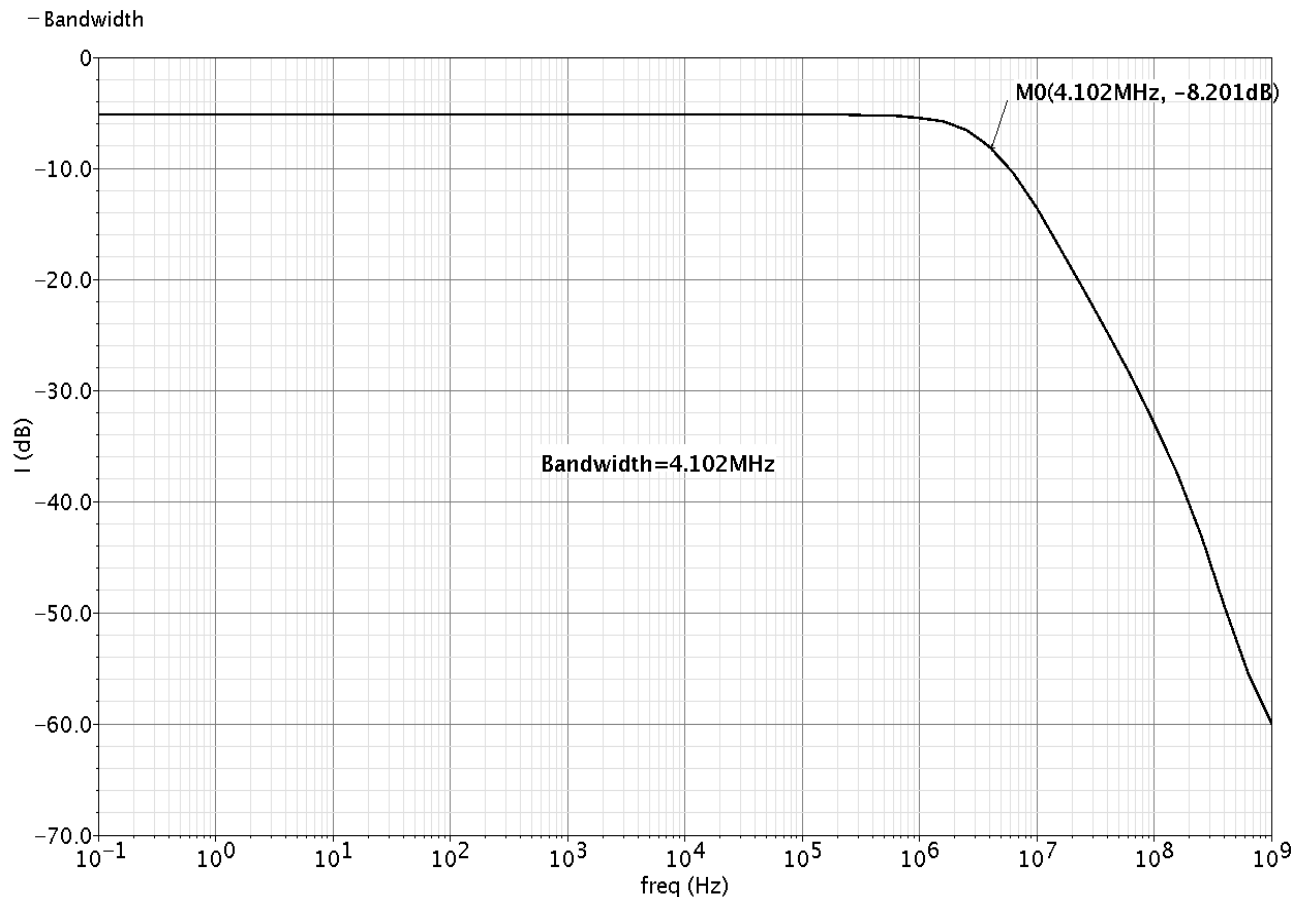

Figure 14. Frequency Response

The performance of the proposed design has been compared with previous works and is being presented in Table 2 . 
International Journal of VLSI design \& Communication Systems (VLSICS) Vol.4, No.2, April 2013

Table 2. Comparison with previous works

\begin{tabular}{|c|c|c|c|c|c|c|}
\hline Parameters & $\begin{array}{l}\text { Proposed } \\
\text { Work }\end{array}$ & REF[12] & REF[7] & REF[9] & REF[8] & REF[4] \\
\hline $\begin{array}{c}\text { Process } \\
\text { Technology }(\mu \mathrm{m})\end{array}$ & 0.18 & 0.18 & 0.50 & 0.25 & 0.50 & 0.50 \\
\hline $\begin{array}{c}\text { Supply Voltage } \\
\text { (Volts) }\end{array}$ & $\begin{array}{l}+1.0 \text { (single } \\
\text { supply) }\end{array}$ & \pm 1.8 & \pm 0.75 & \pm 0.45 & \pm 0.75 & \pm 0.75 \\
\hline $\begin{array}{c}\text { Current } \\
\text { Range }(\mu \mathrm{A})\end{array}$ & $0-1500$ & - & $0.1-500$ & $0-150$ & $0-500$ & $\sim 2-500$ \\
\hline $\begin{array}{c}\text { Output } \\
\text { Impedance }(\mathrm{T} \Omega)\end{array}$ & 1.125 & 0.045 & 0.00135 & 0.0153 & 0.00167 & 0.7854 \\
\hline $\begin{array}{c}\text { Input } \\
\text { Impedance }(K \Omega)\end{array}$ & $\begin{array}{c}3.73\left(\mathrm{I}_{\text {bias }}=\right. \\
50 \mu \mathrm{A})\end{array}$ & 20000 & 0.2 & 1.99 & 0.48 & - \\
\hline $\begin{array}{c}\text { Power } \\
\text { Dissipation }(\mu \mathrm{W})\end{array}$ & 10.56 & 1.5 & - & 96.5 & 1500 & 250 \\
\hline Bandwidth(MHz) & 4.1 & 15.8 & 500 & - & 640 & 631 \\
\hline
\end{tabular}

\section{CONCLUSION}

This paper demonstrates a floating gate MOSFET (FGMOS) based low-voltage, low-power regulated cascode current mirror which exhibits a very high output impedance of $1.125 \mathrm{~T} \Omega$ and dissipates power as low as $10.56 \mu \mathrm{W}$ at an input biasing current of $10 \mu \mathrm{A}$. The current range for the circuit is also at par with respect to other design. The proposed design can work well with low-power, low-voltage analog signal processing \& bio-medical application. Measured performance of proposed current mirror is comparable with other proposals reported to date, as shown in Table 2. The simulation is done in Cadence design environment for $180 \mathrm{~nm}$ CMOS technology with a single power supply of +1.0 Volts.

\section{REFERENCES}

[1] Esther Rodriguez-Villegas, "Low Power and Low Voltage Circuit Design Techniques with FGMOS Transistor”, IET Circuit and Device System Series 20.

[2] Hitesh, Anuj Goel, "Advancement in Current Mirror Technique”, International Journal of Advance Research in Computer Science \& Software Engineering, Vol. 2, Issue 1, January 2012.

[3] Yin, L., Embabi, S. H. K., and Sánchez-Sinencio, E.: "A floating-gate MOSFET D/A converter", Proceedings of the IEEE International Symposium on Circuits and Systems, 1997, pp. 409-12.

[4] Parshotam S. Manhas, Susheel Sharma, K. Pal, L.K.Mangotra \& K.S. Jamwal, "High performance FGMOS-based low volage current mirror", Indian journal of Pure \& Applied Physics, Vol. 46, May 2008, pp. 355-358

[5] Behzad Razavi, Design of analog integrated circuits, McGraw Hill, 2001.

[6] S.S Rajput and S.S Jamuar, "A high performance current mirror for low voltage designs", AsiaPacific Conference on Circuits and Systems, pp. 170-173, Dec 2000.

[7] Susheel Sharma, S.S. Rajput, L.K. Mangotra and S.S. Jamuar, "FGMOS based wide range low voltage current mirror and its application", Asia-pacific conference on Circuit \& Systems. Vol. 2 pp. 331-334, Oct 2002.

[8] Rockey Gupta, Shusheel Sharma, S.S. Jamuar, "A low voltage current mirror based on quasi-floating gate MOSFET", IEEE Conference, 2010.

[9] Bhawna Aggarwal, Maneesha Gupta, "Low-voltage cascode current mirror based on bulk-driven MOSFET and FGMOS Techniques", International Conference on Advances in Recent Technologies in Communication and Computing, 2009.

[10] D.Johns and K.Martin, “Analog Integrated Circuit Design”, John Wiley \& Sons, 1997. 
International Journal of VLSI design \& Communication Systems (VLSICS) Vol.4, No.2, April 2013

[11] P.E. Allen and D.R. Holberg, "CMOS Analog Circuit Design", 2nd ed. Oxford, U.K. : Oxford, 2002.

[12] Seyed Javad Azhari, Khalil Monfaredi, and Hassan Faraji Baghtash, "A Novel Ultra Low Power High Performance Atto-Ampere CMOS Current Mirror with Enhanced Bandwidth”, Journal of Electronics Science and Technology, Vol. 8, No. 3, September 2010.

[13] Costas Laoudias and Costas Psychalinos, "Application of current mirrors in analog signal processing", Physics Department, Electronics Laboratory, University of Patras, Rio Patras, Greece.

Authors

Mr. Abhinav Anand : He received his B.Tech in Electronics \& Telecommunication Engineering from College of Engineering Roorkee, Uttarakhand. He has worked as Lecturer with Uttaranchal Institute of Technology, Dehradun. Currently he is an M.Tech student in VLSI design \& Embedded System in School of Electronics Engineering at KIIT University, Bhubaneswar, Odisha. His main researches focus on Low-Voltage, Low-Power analog circuit design.

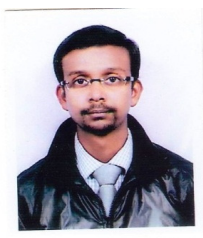

Dr. Sushanta K. Mandal : He received his B.E. degree in Electrical Engineering from Jalpaiguri Govt. Engineering College, West Bengal, India in 1993, MS and Ph.D degree from Indian Institute of Technology (IIT), Kharagpur, India in 2002 and 2008 respectively. He has 12 years of teaching and industry experience. He was actively associated with Advanced VLSI Design Laboratory at IIT. Kharagpur from 2002-2007. From August 2007 to June 2010, he worked as an Assistant Professor at Dhirubhai Ambani Institute of Information and Communication Technology (DA-IICT), Gandhinagar. Currently he is a Professor at KIIT University, Orissa since July 2010. He has published number of research papers in reputed journals and conferences. His research interests include modelling and synthesis of on-chip passive devices, digital, analog and mixed-signal CMOS VLSI design, highlevel synthesis and optimization of analog circuits, soft computing applications in VLSI.

Mrs. Anindita Dash : She received her B.Tech in Electronics \& Telecommunication Engineering from Ghanashyam Hemalata Institute Of Technology and Management, Odisha in 2008. She has worked as embedded engineer in Larsen and Toubro Embedded Division , Mysore from June 2008 to September 2010. Currently She is pursuing M.Tech in VLSI design \& Embedded System in School of Electronics Engineering at KIIT University, Bhubaneswar, Odisha. Her area of research includes low-voltage, lowpower, high speed analog and mixed signal circuit design.

Mr. B. Shivalal Patro : He has completed his B.Tech from Trident Academy of Technology in Electronics \& Telecommunication Engineering in 2010, Odisha. He then completed M.Tech in Communication Systems from KIIT University in 2012. Currently, he is a Ph.D scholar at KIIT University, Bhubaneswar, Odisha. His area of research includes high speed, low power analog and mixed signal IC design and optimization.
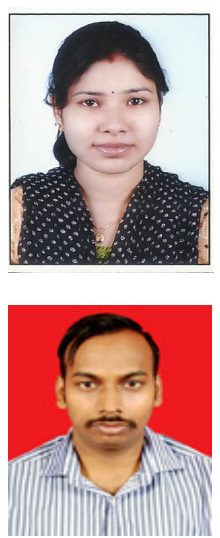